\title{
SATISFACCION DEL USUARIO EN LA EMERGENCIA DEL HOSPITAL CENTRAL DE MARACAY
}

\author{
SATISFACTION OF USERS OF THE EMER- \\ GENCY ROOM AT MARACAY'S CENTRAL \\ HOSPITAL
}

\section{SATISFAÇÃO DE O USUÁRIO NA EMERGÊN- CIA DO HOSPITAL CENTRAL DE MARACAY}

\section{Resumen}

El nivel de satisfacción de los usuarios debe ser visto como un indicador fundamental del Impacto de calidad asistencial. El objetivo fue evaluar la satisfacción del usuario de la Emergencia del Hospital Central de Maracay. Metodología: se realizó una investigación descriptiva de corte transversal con una muestra no probabilística intencional a partir de los usuarios externos e internos del Hospital. Se utilizó como instrumento un cuestionario validado por juicio de expertos y la confiabilidad determinada por Alfa de Combrach de 0,86. Resultados

Gómez G.Wuilman E. ${ }^{1}$

Dávila L. Fanny J. ${ }^{2}$

Campins R. Rafael A. ${ }^{2}$ Colmenarez D. Stefanny ${ }^{3}$

${ }^{1}$ Dirección General de Salud Ambiental-MPPS.MaracayVenezuela.

2IAE "Dr. Arnoldo Gabaldon" - MPPS. Venezuela. MaracayVenezuela.

${ }^{3}$ Facultad de Medicina. Universidad de Carabobo.

Trabajo recibido: 18 de Noviembre de 2016. Aprobado: 01 de Agosto de 2017. Según el usuario externo la calidad de atención se relaciona con las variables limpieza y orden (58\%), seguridad (48\%), equipos médicos quirúrgicos (57\%), tiempo para ser atendido (48\%), trato de personal médico (42\%) y de enfermería (48\%) influyeron de manera determinante en la percepción positiva de la calidad de atención. Mientras que para el usuario interno las variables relacionadas a calidad de atención fueron el espacio físico y la distribución de los mismos (42\%), la limpieza y orden $(60 \%)$ y la capacitación que recibe el personal de salud (33\%). Conclusiones: El análisis proporciona información válida para generar respuestas oportunas y optimizar los recursos sanitarios en el Hospital Central de Maracay.

Palabras clave: Salud publica, Satisfacción del Usuario, Calidad asistencial. 
Introduction: The users' level of satisfaction must be seen as an essential indicator of the impact of care quality. The objective was to evaluate the satisfaction of the user of the Emergency Room at Maracay's Central Hospital. Methodology: Descriptive crosssectional research was performed with an intentional no probability sample from internal and external hospital users.A questionnaire validated by experts' judgment was used and reliability of 0.86 determined by Combrach's Alpha. Results: According to the external user, attention quality is related to the variables of cleanliness and tidiness (58\%), safety (48\%), medical team (57\%), waiting time (48\%), medical staff behavior (42\%); and nurses behavior (48\%) which greatly influenced the positive perception of attention quality. Whereas for the internal user the variables related to attention quality were physical space and distribution (42\%), cleanliness and tidiness (60\%) and training received by health care staff $(33 \%)$. Conclusions: The analysis offers valid information to generate convenient answers and optimize health resources at Maracay's Central Hospital.

Key words: Public health, User's Satisfaction, Care quality.

\section{Resumo:}

Introdução: o nível de satisfação dos usuários deve ser visto como um indicador fundamental do impacto da qualidade dos cuidados. O objetivo foi avaliar a satisfação do usuário do Hospital Central de Emergência Maracay. Metodologia: uma pesquisa descritiva transversal foi realizada com uma amostra intencional não-probabilística dos usuários externos e internos do Hospital. Um questionário validado por julgamento de especialistas e a confiabilidade determinada pelo Combrach Alpha de 0,86 foi usado como instrumento. Resultados De acordo com o usuário externo, a qualidade dos cuidados está relacionada às variáveis de limpeza e ordem (58\%), segurança (48\%), equipamento médico cirúrgico (57\%), tempo a ser atendidos (48\%), 42\%) e enfermagem (48\%) tiveram influência decisiva na percepção positiva da qualidade dos cuidados. Enquanto para o usuário interno as variáveis relacionadas à qualidade de atendimento foram espaço físico e distribuição (42\%), limpeza e ordem (60\%) e treinamento recebido pelo pessoal de saúde (33\%). Conclusões: a análise fornece informações válidas para gerar respostas oportunas e otimizar recursos de saúde no Hospital Central de Maracay.

Palavras-chave: Saúde pública, Satisfação do usuário, Qualidade de atendimento.

\section{Introducción}

La crisis de los servicios de salud en Venezuela ha producido una gran insatisfacción de la población ante la oferta y la calidad de estos, evidenciándose, una desarticulación institucional herencia de un inadecuado modelo de financiamiento y de gestión, deficiente participación de la sociedad civil, obsoleto modelo de atención y deficiencia en lo que respecta a calidad y acceso $(1 ; 2 ; 3 ; 4)$.

Existe una estrecha relación entre calidad de atención y satisfacción de los usuarios para lograr el equilibrio del Sistema de Salud. La calidad de la atención en salud es un tema que adquiere cada vez más relevancia debido a la libertad con que opinan los usuarios acerca de los servicios recibidos, de los encargados de prestar servicios de salud y de las propias instituciones proveedoras de estos servicios. Además la calidad de atención es condicionada por los retos organizacionales y la creciente presión social determinada por la toma de conciencia de una sociedad más informada acerca de sus derechos. En términos generales, la satisfacción en salud se refiere a tres aspectos: organizativos (tiempo de espera, ambiente); atención recibida y su repercusión en el estado de salud de la población, 
y el trato recibido durante el proceso de atención por el personal implicado (3). La calidad se consigue no solo diseñando, ejecutando y evaluando el proceso tecnológico (calidad intrínseca) sino también modificando la impresión que los usuarios tiene sobre la idoneidad de un producto para satisfacer sus expectativas, es decir, la calidad percibida $(4 ; 5 ; 6 ; 7)$.

Otros autores han señalado la importancia y la pertinencia de introducir la satisfacción de los usuarios con los servicios de salud como parte y complemento de otras actividades de control de calidad. $(8 ; 9 ; 10 ; 11 ; 12)$

En los Servicios de Urgencias o Emergencias hospitalarias se hace necesario, para la mejora continúa de la calidad, conocer qué opinan los clientes e incorporar esta visión a la evaluación de las tareas, por ser este nivel el primer contacto con el sistema sanitario. Este método representa una de las formas más rápidas para evaluar algunos aspectos de la calidad de los servicios y ofrece beneficios a un costo relativamente bajo (13).

De los usuarios se obtiene la información acerca del desempeño de los servicios públicos y se logra determinar en qué medida los servicios satisfacen sus necesidades y expectativas por ello en la búsqueda de realizar investigaciones pertinentes, integrales y en repuesta a las necesidades de la población se planteo desarrollar la evaluación de la calidad para el mejoramiento de la gestión en la emergencia del Hospital Central de Maracay (HCM).

La Organización Panamericana de la Salud (14) define como Funciones Esenciales de la Salud Pública (FESP) al conjunto de actuaciones que deben ser realizadas con fines concretos, necesarios para la obtención del objetivo central, que es así mismo la finalidad de la salud pública, es decir, mejorar la salud de las poblaciones. Entre otras destaca para los fines de este trabajo la FESP 9 es decir la referida a la "Garantía y mejoramiento de la calidad de los servicios de salud individuales y colectivos". Esta FESP a su vez está definida por la promoción de la existencia de los sistemas de evaluación y el mejoramiento de su calidad, el fomento de la elaboración de normas sobre las características básicas que deben tener los sistemas de garantía y mejoramiento de la calidad y supervisión del cumplimiento de los proveedores de servicios que tengan esta obligación, así como, la definición, explicación y garantía de los derechos de los usuarios.

En Venezuela la Constitución de la Republica Bolivariana de Venezuela (15) en su artículo 83, establece que "la salud es un derecho social fundamental, obligación del Estado, que lo garantizará como parte del derecho a la vida" y el Ministerio del Poder Popular para la Salud (MPPS) como ente rector del Sistema Público Nacional de Salud (SPNS) debe implementar los mecanismos necesarios para garantizar a los ciudadanos y ciudadanas, la promoción, la defensa y la recuperación de su salud a nivel individual y colectivo. Para ello, las actividades de investigación son esenciales para aumentar la eficiencia del sistema público nacional de salud. En tal sentido, es necesario fortalecer la gestión de la red de servicios de salud a nivel local, regional y nacional, ya que existe una ausencia de mecanismos de control y monitorización de las políticas, ausencia de indicadores de evaluación pertinentes y la necesidad de organizar el SPNS.

En virtud de lo expuesto y de que la investigación en salud debe ser desarrollada en función de las necesidades y de las políticas en salud dirigidas hacia la disminución de las brechas sociales e inequidades, y para aportar con un esfuerzo de búsqueda de respuestas pertinentes, integrales y sustentables a las necesidades de la población. Por otra parte, con el desarrollo de la humanidad, y en particular con los avances en el terreno de la medicina, es necesario hacer un llamado para humanizar la asistencia en salud y ofrecer servicios de mayor calidad. La calidad en la atención médica debe estar basada en actividades encaminadas a garantizar los servicios de salud accesibles y equitativos con profesionales óptimos y teniendo en cuenta los recursos disponibles, logrando la satisfacción del usuario con la atención recibida.

Los Servicios de Emergencia son considerados como la zona donde se percibe la mayor insatisfacción por parte de los usuarios, en el caso de los usuarios de hospitales se produce una espera prolongada por atención de salud y camas, existe una desconfianza ante el 
personal por experiencias propias o de personas cercanas como familiares y amigos en que se reconocen experiencias de mal trato.

Esta investigación no pretende invertir el orden de las prioridades asistenciales, pero sí busca ayudar a resolver el difícil reto de "saber responder a esas necesidades de salud siendo capaces de satisfacer, también, las expectativas de los pacientes". El enfoque de los cuidados orientados hacia el paciente no pretende otra cosa que satisfacer ambos objetivos. El objetivo de la presente investigación busca evaluar la calidad para el mejoramiento de la gestión en el área de emergencia del Hospital Central de Maracay.

\section{Material y Método}

El estudio propuesto se corresponde a una investigación no experimental descriptiva de corte transversal.

El universo estuvo comprendido por:

a) Los usuarios internos del área de emergencia del Hospital Central de Maracay (Médicos, Enfermeras y auxiliares de enfermería)

b) Los usuarios externos o personas atendidas en el área de emergencia del Hospital Central de Maracay y/o familiar acompañante.

Se trabajó con una muestra No Probabilística Intencional o de Conveniencia, dada por 100 usuarios internos y 100 usuarios externos, para lo cual se fijaron los siguientes criterios:

- Criterios de Inclusión: toda persona mayor de 18 años y menores de 66 años que se encuentre recibiendo atención en el área de emergencia o familiar - acompañante y trabajador con mas de 3 años laborando en la institución

- Criterios de Exclusión: evidencia de enfermedad o patología psiquiátrica o retardo mental. Personas menores de 18 años o mayores de 66 años.

La técnica utilizada fue la encuesta y el instrumento utilizado fue un cuestionario dirigido a la población en estudio, diseñado por los autores de la investigación al cual se precisó la validez por juicio de expertos en el área de Gestión de Hospitales, Estadística y Gestión en Salud Pública, mientras la Confiabilidad se obtuvo según fórmula Alfa de Combrach con un valor de 0,86 . Este instrumento constó de tres partes: Datos personales, datos específicos (que contienen 21 preguntas cerradas) que abordaron la dimensión usuario externo (12 ítems), usuario interno (9 ítems). En cada dimensión se abordo aspectos humanos, técnica y entorno. El instrumento de medición tuvo una longitud de 22 ítems y una amplitud de escala de cuatro categorías de excelente, bueno, regular y nunca, respectivamente.

Partes del instrumento de recolección de datos:

Parte I. Datos generales: Aborda los aspectos relacionados con la edad, sexo, grado militar, número de consultas en el presente año y servicio donde fue atendido.

Parte II. Datos específicos: Aborda la calidad de la atención de salud considerando

-Dimensión Humana (03)

-Dimensión Técnico-científica (11)

-Dimensión del Entorno (08)

Se utilizó la estadística descriptiva como técnica de análisis de datos, utilizando frecuencias y porcentajes simples, los cuales permitieron presentar de manera detallada los resultados obtenidos en forma de cuadros y gráficos, representando los resultados valores absolutos y relativos. Los investigadores realizaron las encuestas los días lunes y viernes del mes de Julio del año 2012 en el siguiente horario:

a) Lunes: de 7:00 am a 1:00 pm.

b) Viernes: de 1:00 pm a 7:00 pm 


\section{Resultados}

\section{Situación de la Emergencia del Hospital Central de Maracay}

La emergencia de adultos del HCM dispone de 44 camas las cuales están distribuidas 16 camas en observación y 28 en área Central. En relación al suministro de tratamiento a los usuarios, no hay capacidad para cubrir el $100 \%$ de lo indicado. En cuanto a los insumos, se dispone de una dotación mínima necesaria para la atención de los usuarios, según lo establecido por cada jefatura de los departamentos, en conjunto con central de suministro quedando registrados en formatos por escrito para dicho fin.

Hay una coordinación general de Enfermería con su adjunto, quienes cuentan con un equipo de enfermeras profesionales, encargadas de supervisar los diferentes departamentos el cumplimiento de las actividades previstas. Constituye una de las puertas de entrada para los usuarios en tres Servicios manejados en el Hospital los servicios de: Medicina Interna, Cirugía y Traumatología.

El personal de enfermería rota en tres turnos: Mañana, tarde y noche, con una distribución de asignación por camas de 2:1 en el área de Observación y de 6:1 en el área de Central.

La distribución de las áreas es la siguiente (Fig. 1 y 2):

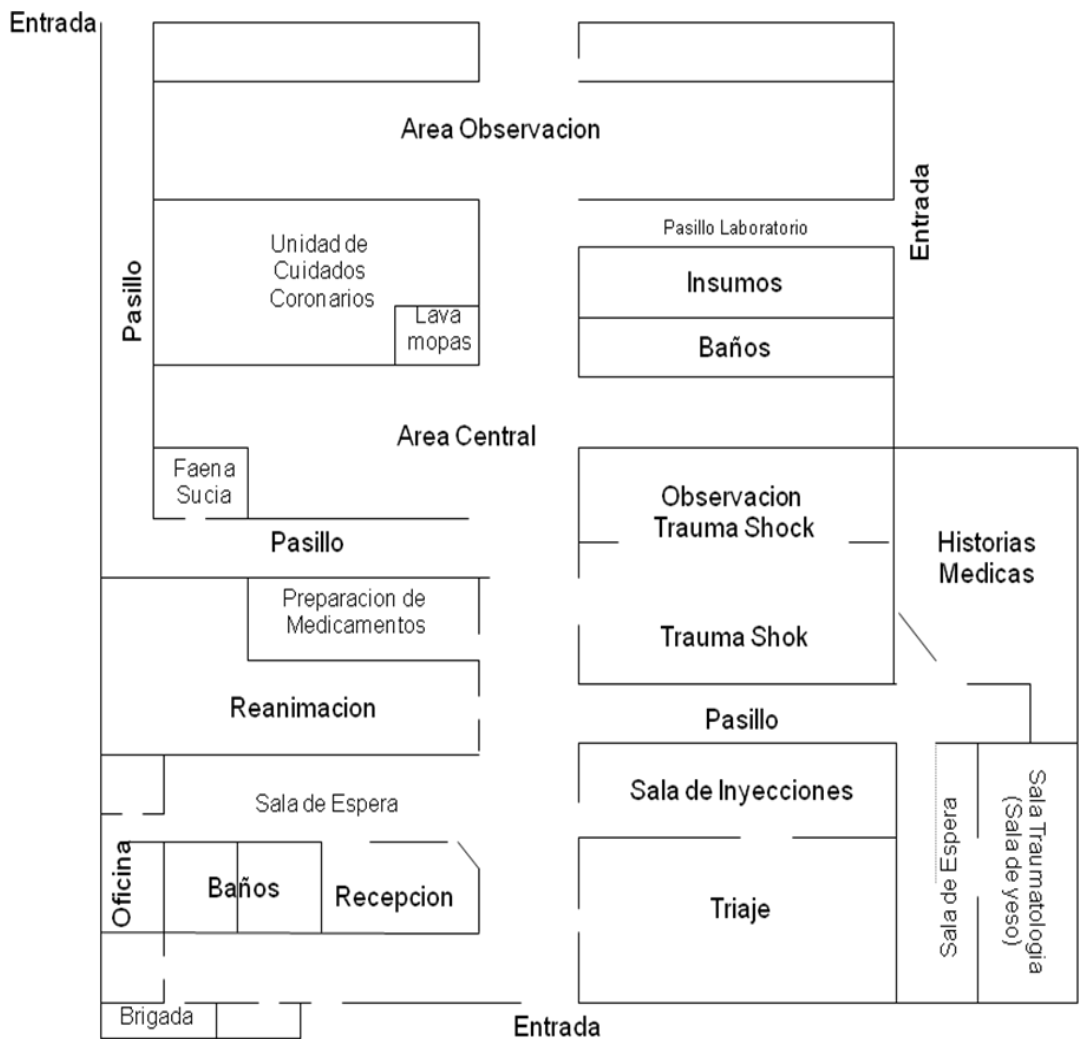

Fig $\mathrm{N}^{\circ}$ 1: Mapa de distribución de las áreas de la Emergencia de Adultos de Hospital Central de Maracay 


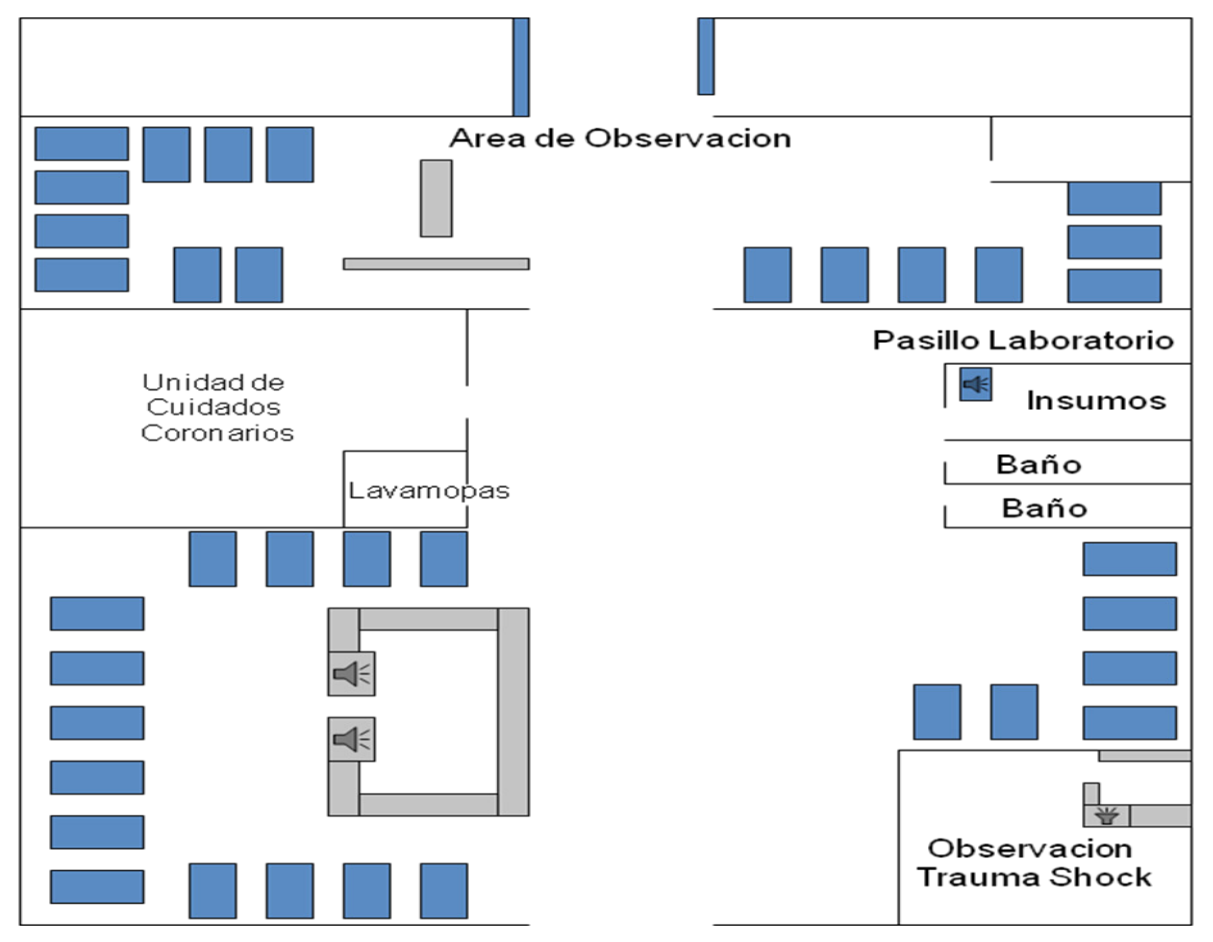

Fig N²: Mapa de Distribución del Área Central del área de la Emergencia de Adultos de Hospital Central de Maracay

La emergencia de adulto cuenta con la presencia de residentes de postgrado, así como, especialista en Cirugía y Medicina Interna en las áreas de consulta de pacientes, un área de pabellón, sala de yeso, sala de reanimación y las áreas de observación.

\section{Características y percepción del usuario externo}

La encuesta se realizo en la Emergencia de Adultos del Hospital Central de Maracay los días lunes en la mañana y viernes en la tarde del mes de julio del año 2011. Se consideraron usuarios externos pacientes u acompañante del mismo, que una vez explicado el alcance y objetivos de la investigación estuviese dispuesto a responder a las preguntas.

De todas las personas encuestadas $70 \%$ fueron mujeres tal vez motivado a que las mujeres asisten generalmente como acompañantes de algún familiar enfermo. Se determino que en la muestra la mayoria de los encuestados provenian de Aragua (88,44\%), seguido en orden de importancia por los procedentes del estado Miranda $(6,18 \%)$, Guarico $(3,22 \%)$ y el estado Carabobo (2,15\%).

Cuando se identifico el nivel de instrucción de los usuarios contemplados en la muestra se observo que $23,66 \%$ tenían la primaria completa y $22,85 \%$ la secundaria completa.

Según Grafico 1 se observa que un $40 \%$ de los usuarios consideran que las instalaciones del Área de Emergencia son Regulares y un 39 \% las clasifica como buenas. (Ver X.1 )

De igual forma, al preguntar por el espacio físico y la distribución de los mismos 56,5\% los considera regulares. (Ver X.2)

Muy bien ponderada estuvo la limpieza y orden en el área de Emergencia al obtener $50 \%$ de usuarios que la clasificaron como Buena y $5 \%$ como Excelente. (Ver X.3)Por el contrario el confort en el área es visto por $47 \%$ de los encuestados como Malo y un 34\% lo considera regular. (Ver X.4) El aspecto de la Seguridad en la Emergencia de adultos 
del Hospital Central de Maracay fue clasificado como Bueno por $48 \%$ de los encuestados. (Ver X.5)

En cuanto a los equipos médicos quirúrgicos con que cuenta el área de emergencia 57\% considera que son Buenos. (Ver X.6) no obstante la dotación de insumos y materiales médico-quirúrgicos fue mencionada como de regular (37\%) a Mala (12\%). (Ver X.7)

Los usuarios consideran que el tiempo para ser atendidos es excelente con menos de 1 hora de espera (47\%). (Ver X.8) Los usuarios de la Emergencia consideran que la información suministrada por el personal en la atención es de mala (14,5\%) a regular (45\%). (Ver X.9) Cuando se pondera el trato del personal médico al usuario este es considerado como de Bueno (42\%) a Excelente (26\%) mientras que el trato del personal de enfermería es catalogado de similar manera como de Bueno (48\%) a Excelente (31\%). (Ver X.10 y X.11)

Finalmente la calidad de atención en General en la Emergencia de Adultos del Hospital Central de Maracay fue denominada como de Regular (42\%) a Buena (39\%). (Ver X.12)

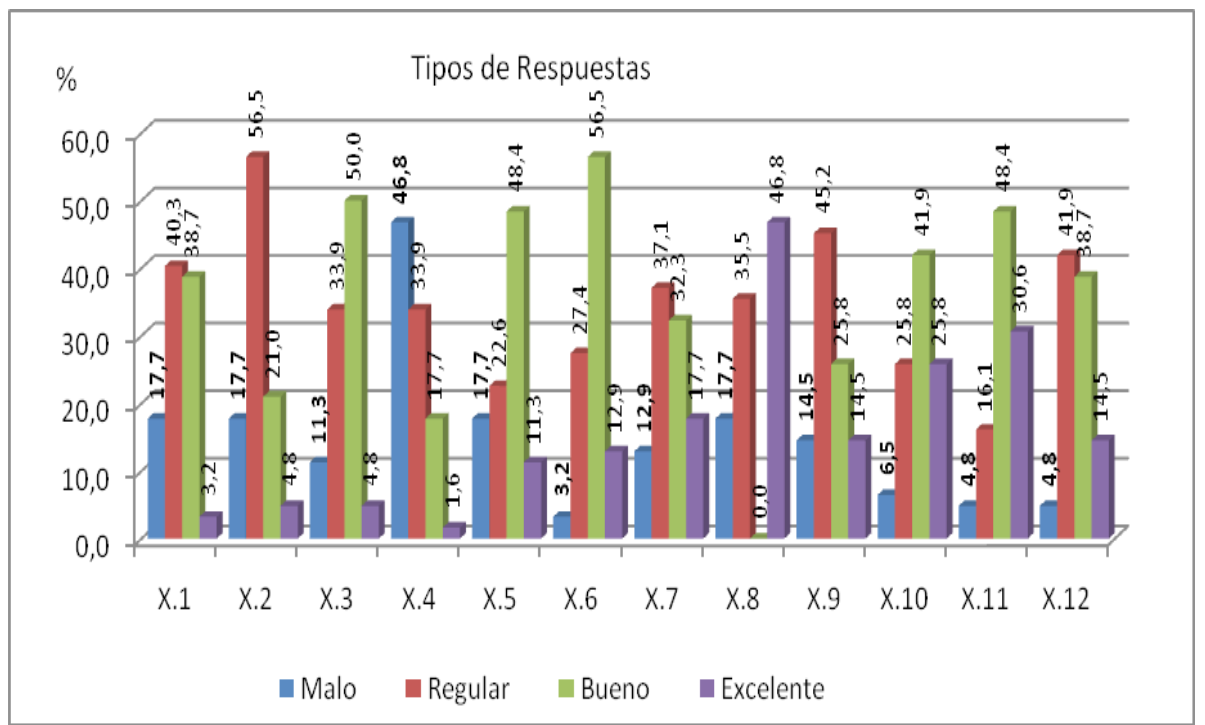

Grafico $N^{0} 1$ : Distribución porcentual de respuestas a encuesta satisfacción en usuario externo en el Hospital Central de Maracay. 2011.

Leyenda: X.1.Instalaciones, X.2 Espacio fisico y distribución, X.3 Orden y Limpieza, X.4 Confort, X.5 Seguridad, X.6 Equipos Médicos Quirurgicos, X.7 Insumos y materiales, X.8 Tiempo de atención, X.9 Información, X.10 Trato del médico, X.11 Trato de enfermería, X.12 Calidad de atención.

\section{Características y percepción del usuario interno}

Se consideraron usuarios internos personal médico y de enfermería que labora en la Emergencia de adultos del Hospital Central de Maracay a los cuales una vez explicado el alcance y objetivos de la investigación estuviese dispuesto a responder a las preguntas.

De 100 personas encuestadas durante el mes de julio de $201183 \%$ fueron del sexo femenino. Respecto a los rangos de edad de los encuestados entre los usuarios internos la mayoría está entre los 31 a 45 años (51\%). En relación al nivel de instrucción de los encuestados en su mayoria tiene como nivel de instrucción Tecnico Universitario (54\%). Un aspecto interesante es que muy poco personal del HCM encuestado tiene cargo fijo (21), $44 \%$ estaban en condición de suplentes y $35 \%$ Contratado.

Según Gráfico $\mathrm{N}^{\circ} 2$ se evidencia que hay coincidencia en un $42 \%$ de los usuarios que consideran que el espacio físico y la distribución de los mismos es Regular y Bueno, mientras que un $16 \%$ las considera como Malo. (Ver X.1) 
$\mathrm{Al}$ preguntar por la limpieza y el orden en el área de emergencia el $60 \%$ de los usuarios le refiere como Bueno (Ver X.2). En lo que respecta a la seguridad el 57\% de los usuarios la clasificaron como Regular, mientras que un 30\% la considera Mala y un 13\% Bueno (Ver X.3)

En lo referente a los equipos medico-quirúrgicos del área de Emergencia del Hospital Central de Maracay los resultados fueron que un $78 \%$ de los usuarios los clasifico como regular y un $13 \%$ de los encuestados los clasifico como Malo (Ver X.4) En cuanto a la dotación de insumos y materiales médicos quirúrgicos en esta área, el $66 \%$ de los usuarios consideran que es regular, un 26\% que es Mala y un $8 \%$ la englobo como Buena. (Ver X.5) En relación a la cantidad de personal para laborar en el área de emergencia un $43 \%$ de los usuarios internos de la Emergencia la consideran Mala, un 43\% Regular y un 14\% Buena. (Ver X.6) Cuando se pondera la relación número de paciente vs. Numero de personal para atenderlo, es considerada como Mala por un $52 \%$ de los encuestados, Regular por un $38 \%$ y Buena un $10 \%$. (Ver X.7

El aspecto capacitación que recibe el personal de salud fue clasificado como Malo por un $34 \%$ de los Usuarios, Bueno por un 33\%, Regular por un 34\% y Excelente solo un 9\% de los encuestados. (Ver X.8)

Para finalizar, en el ítem cómo calificaría la calidad de atención del personal al usuario del área de emergencia de Adultos del Hospital Central de Maracay los resultados fueron Buena 52,\%, Regular 32\% y Excelente 16\% ( Ver X.9)

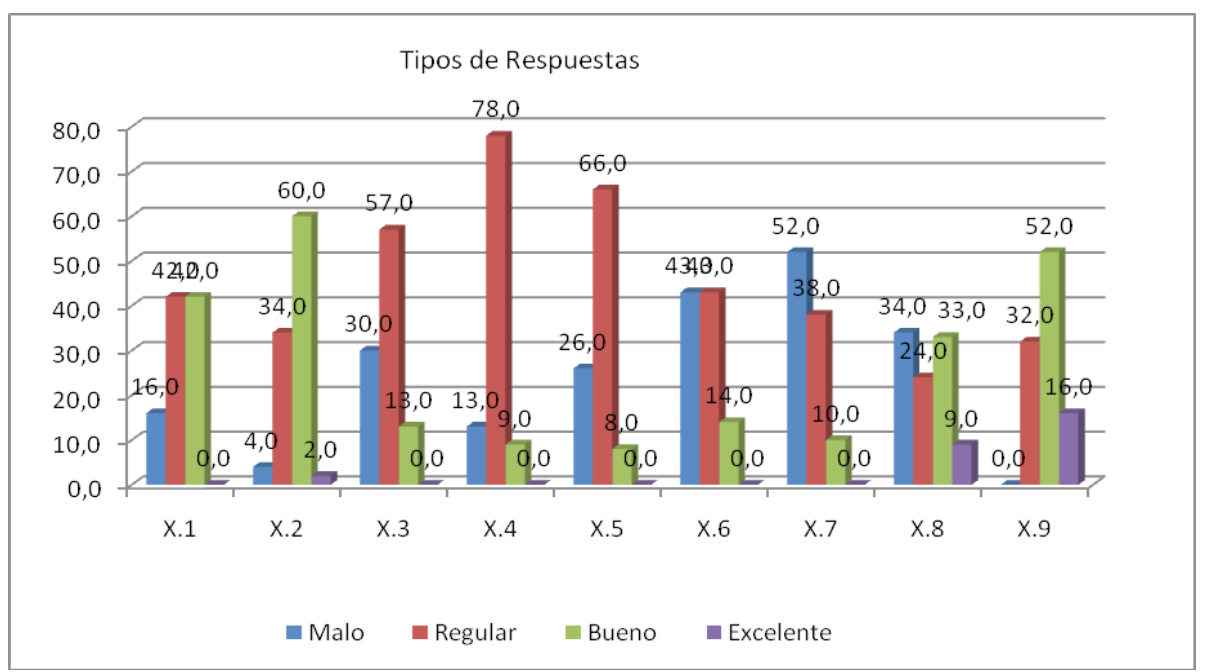

\section{Gráfico N²: Distribución porcentual de respuestas a encuesta satisfacción en usuario} interno en el Hospital Central de Maracay. 2011.

\section{Leyenda:}

X.1 Espacio fisico y distribución, X.2 Orden y Limpieza, X.3 Seguridad, X.4 Equipos Médicos Quirurgicos, X.5 Insumos y materiales, X.6 Cantidad de personal, X.7 Proporcion paciente y personal de salud, X.8 Capacitación del personal, X.9 Calidad de atención del personal al usuario.

\section{Discusión}

En este trabajo se evalúa la opinión del usuario externo e interno y no se explora la dimensión técnica, la productividad, ni el desempeño.

Los resultados obtenidos con respecto al Usuario Externo muestran el predominio de la variable sexo femenino con $70 \%$, tan alta como los observados en trabajos de Córdoba, Argentina donde también estuvo a favor de este sexo con $85 \%$, hecho que se repite con 
igual porcentaje en el trabajo de Hidalgo, México $(16 ; 17 ; 18)$.

En el Hospital Central de Maracay la mayoría de los usuarios cataloga las Instalaciones y espacio físico como regulares, pero el confort fue catalogado de regular a malo, hechos que contrastan con la literatura revisada. Sin embargo la limpieza y orden fue calificada de buena a excelente. Cuando se coteja estos puntos con otros estudios revelan que las instalaciones, el confort y la apariencia son los aspectos que reflejan mayor fragilidad en los centros de atención. $(16 ; 19)$.

En HCM mas del $50 \%$ de los usuarios consideraron buenos los equipos médicos quirúrgicos, mientras la dotación de insumos y materiales quirúrgicos fue catalogada de regular a mala lo que contrasta con los hallazgos en Córdoba (16). Los resultados de este trabajo revelaron que para la mayoría de los destinatarios no hay insumos para la atención médica en el HCM.

El Tiempo de espera detectado fue en casi de 50\% considerado menor a una hora .Estos resultados están por debajo de lo encontrado en otros estudios en las comunidades de Córdoba $(100 \%)$ y Lima-Callao $(79,5 \%)$. (16; 19). Este tiempo de espera fue considerado por los usuarios externos del HCM como excelente.

Respecto a la Calidad de información suministrada en la muestra consultada consideran que la misma es de mala a regular en más de un $50 \%$ muy por encima de lo evidenciado por otros estudios $(16 ; 17$ y 19$)$.

Según Acosta entre los conceptos asociados a la calidad de atención cobran importancia los aspectos relativos a trato personal e información recibida. La relación profesional y destinatarios de los centros de salud es uno de los puntos considerados importantes para evaluar la satisfacción de usuarios.

En el HCM, este estudio reflejó que los encuestados manifestaban mayor satisfacción con el personal médico y de enfermería, similar a lo evidenciado por Acosta, Bolaños y Ortiz (16), $(17 ; 19)$. De igual manera, los usuarios externos encuestados Calidad de atención en el HCM fue ponderada de regular a buena hallazgo similar a los de Lima-Callao e Hidalgo-México de los autores anteriormente mencionados.

Los resultados obtenidos con respecto al Usuario Interno muestran que el sexo predominante en este trabajo fue el femenino con $83 \%$, con una media de edad que oscila entre los rangos de 31-45 años. Respecto al grado de instrucción se identifico que mayoritariamente presentaban nivel Técnico y en cuanto a su situación laboral solo $21 \%$ posee cargo fijo. En el Hospital Central de Maracay los usuarios internos catalogan las Instalaciones y espacio físico como regular y bueno mientras que para la mayoría del usuario externo la percibió como regular. Respecto a la limpieza y orden fue calificada de buena es decir un nivel de satisfacción similar al del usuario externo.

No obstante la Seguridad en el Área de Emergencia es vista por el usuario interno como de regular a mala en tanto que el usuario externo la percibe como buena. Tal vez el tiempo de permanencia en el área, la rotación de horarios haga que la visión de ambos usuarios sea tan diferente.

Estudios que evalúan la calidad de la atención desde la perspectiva de los usuarios indican que la suficiencia del material y equipo son aspectos importantes para calificar como aceptable un servicio $(16 ; 19)$. En el HCM casi un $80 \%$ de los usuarios internos consideraron regulares los equipos médicos quirúrgicos, similar percepción presentaron de la dotación de insumos y materiales quirúrgicos. Al contrastar con el usuario interno tienen igual percepción para los insumos pero discrepan en lo referente a equipos médicos cuando el usuario interno lo declara como bueno.

En atención a Donabedian (11) el concepto de calidad en el ámbito de la asistencia sanitaria se refiere a la capacidad que puede tener una organización para satisfacer la necesidad de los consumidores de los servicios de salud. A partir de esto puede definirse como un indicador de calidad la satisfacción del usuario, la cual se define como una evaluación positiva por parte del usuario de las diferentes dimensiones que configuran la atención 
sanitaria. En el HCM aspectos como la cantidad de personal necesaria para laborar en el área de emergencia y la relación número de paciente /número de personal fue calificada por el usuario interno de regular a malo. Mientras que la capacitación que recibe el personal de salud fue calificada por el usuario interno de buena a excelente

En el HCM, este estudio reflejó que los usuarios internos cualificaban la calidad de atención al usuario en su mayoría como buena, en tanto, que el usuario externo la observa como regular.

Se concluye al observar los aspectos positivos y negativos de la atención y caracterizar los conceptos de buena y mala calidad con respecto a ella, se hace hincapié en las variables que influyeron en el proceso mismo de la atención emergencia. Según el usuario externo la calidad de atención se relaciona con las variables limpieza y orden, seguridad, equipos médicos quirúrgicos, tiempo para ser atendido, trato de personal médico y de enfermería influyeron de manera determinante en la percepción positiva de la calidad de atención. Mientras que para el usuario interno las variables relacionadas a calidad de atención fueron el espacio físico y la distribución de los mismos, la limpieza y orden, así como, la capacitación que recibe el personal de salud.

Es importante rescatar:

a) La necesidad de un personal calido, preocupado y humano, rescatando la calidez como Valor.

b) Es importante suministrar información expedita con lenguaje claro y directo.

c) Suministrar rapidez en la atención.

El análisis de la calidad de atención desde la óptica de la satisfacción del usuario es importante para proporcionar información válida para generar respuestas oportunas y optimizar los recursos sanitarios en el Hospital Central de Maracay.

Agradecimientos

Al Instituto de Altos Estudios "Dr. Arnoldo Gabaldon" del Ministerio del Poder Popular para la Salud, que prestó su apoyo para el desarrollo de esta investigación.

\section{Bibliografía}

1. Otero, M. J. y Otero I., J. I. Gerencia en odontología. Tomo 2 .Ética y Marketing .Edit. Odontomarketing . Lima, Perú.2002

2. Massip, P.C. ; Ortiz Reyes, R.M.; Llantá Abreu, M.C.; Peña Fortes, M.; Infante Ochoa, I. La evaluación de la satisfacción en salud: un reto a la calidad. Disponible en: http://scielo.sld.cu/scielo.php?script=sci_arttext\&pid=S086434662008000400013\&lng=es. [citado 11 Mar 2014]

3. Medina-Lezama, M. y Esquincha-Medina, A. .Grado de Satisfacción de los Pacientes asegurados hospitalizados en la Clínica de las Mercedes, Hospital Militar de Lima. Rev. Enf. Herediana. 6(2):96-106.2013

4. Banegas Cardero, J. E. Nivel de Satisfacción de los pacientes atendidos en el Servicio de urgencia del Centro Diagnostico Integral "Los Arales". Rev. MEDISAN 18 (7) 942.2014.

5. Sihuin-Tapia,E Y.; Gomez-Quispe,O.E. y Ibáñez-Quispe, V. Satisfacción de Usuarios hospitalizados en un Hospital de Apurimac, Perú. Rev. Perú Med Exp Salud Pública.32 (2): 299-302

6. Lobos A., C. F.; Lóezar, C. ;González V. y Ortega A. Satisfacción con la atención Médica de pacientes hospitalizados en un servicio cínico docente y en uno no docente. Rev. Chil. Salud Pública Vol. 16(1):16-25.2012

7. Varo J. Gestión Estratégica de la Calida de los Servicios Sanitarios. Un modelo de gestión Hospitalaria. Ed. Díaz de Santos. Madrid-España .1994

8. Vuori, H. El control de calidad en servicios sanitarios. Concepto y metodología, $1^{\text {a }}$ 
ed. Barcelona: Masson, Barcelona.1988

9. Ware JE, Davis-Avery A. y Stewart A.L. The measurement and meaning of patient satisfaction. Health Med Care Service Rev 1:1.1978

10. Suñol R.; Pacheco M.V.; Delgado, R; Corbella, A. y Baures, N. El estudio de la opinión del usuario y su aplicación en los programas de control de calidad. Control de calidad asistencial 2:15-22.1987

11. Donabedian A. Evaluación de la calidad de la atención médica. En: OPS/OMS, editores. Investigaciones sobre servicios de salud: una antología. Washington D.C.: OPS/OMS; p.382-404. (Publicación Científica; 534).1992

12. Godínez, F.; Reyes, R.; Arizam, F.; Maruris, M. ; Cuevas, A. y Cabañas, A. Nivel de satisfacción de usuarios de consulta externa en un hospital público. Psicología y Salud, Vol. 24, Núm. 1: 97-107.2014

13. Bronfman; M.J. Del «cuánto» al «por qué»: la utilización de los servicios de salud desde la perspectiva de los usuarios. Salud Publica Mex 39:442-450. 1997

14. Organización Panamericana de la Salud. "La salud Publica en las Américas". Washington D.C.2002

15. Asamblea Nacional de la República Bolivariana de Venezuela. Constitución Nacional de la República Bolivariana de Venezuela. Caracas, Venezuela. Gaceta Oficial Extraordinaria $\mathrm{N}^{\circ} 5.908$ del 19 de febrero de 2009

16. Acosta, L.; Burrote, M.S.; López, M.J.; Lúchese, M.; Cornetto, C.;Ciuffolini, B. y otros .Análisis de la Satisfacción del usuario en centros de Salud del primer nivel e atención en la provincia de Córdoba, Argentina. Rev. Enfermería Global. Vol. 21.pp $1-13.2011$.

17. Ortiz, R.; Muñoz, S.; Martín, D. y Torres, E. Consulta externa en instituciones de Salud de Hidalgo, México, según la opinión de los usuarios. Rev. Panam. Salud Pública.13 (4) 229-238.2003

18. Morillo, J. y Aular, Y. Satisfacción del Cliente de la Consulta Externa de Pediatría en Clínicas Privadas. Rev. Venezolana de Gerencia.5 ( 52): 591-603.2010

19. Bolaños, G.; Mongrut, U.; Morales, M.; Muñoz, E.; Sagastegui, A. y Toribio, R. Calidad de atención y satisfacción del usuario en los centros de referencia de enfermedades de Transmisión sexual de Lima y Callao. Rev. Salud, Sexualidad y Sociedad 2 (3) .2009) 